\title{
COMPOSICIÓN, DINÁMICA Y ABUNDANCIA DE LA COMUNIDAD FITOPLANCTÓNICA DE UNA LAGUNA CON AGUAS DE ORIGEN VOLCÁNICO
}

\author{
MARGARITA SALAZAR-SÁNCHEZ ${ }^{1}$ \\ GERARDO NAUNDORF-SANZ1 \\ MARIO MEDINA-TOMBÉ ${ }^{1}$ \\ margaritasalaz@gmail.com
}

Manizales, 2011-06-03 (Rev. 2011-06-29)

\section{RESUMEN}

Se presenta la composición y dinámica del fitoplancton de la laguna Aguas Tibias, Coconuco, Puracé. Se realizaron muestreos mensuales durante octubre de 2006 a junio de 2007. Se midieron temperatura, $\mathrm{pH}$, oxígeno disuelto, conductividad y nutrimentos ( $\mathrm{N}$ y $\mathrm{P}$ ). El fitoplancton se identificó y se cuantificó. Se encontraron 55 géneros, los Diatomophytas fueron los más abundantes, siguiéndole en importancia Cyanophyta, Euchlorophyta y Zygophyta. En la laguna Aguas Tibias los géneros Tabellaría (19,25\%), Anomoeoneis $(10,45 \%)$, Navícula $(7,73 \%)$ y Chlamydomona $(7,33 \%)$ fueron los más abundantes en la columna de agua, a diferencia de los géneros Selenastrum (0,06\%), Gyrosigma $(0,01 \%)$, Xantidium $(0,02 \%)$, con escasa presencia. Las variaciones detectadas no son significativas estadísticamente, tanto en función de espacio (profundidad) como tiempo (meses de muestreo). Se encontraron valores muy altos para calcio, alcalinidad, dureza cálcica y total y conductividad, debido en parte al origen volcánico del cuerpo de agua. Con base en los géneros de algas encontrados, se propone la laguna como un ecosistema mesotrófico.

\section{PALABRAS CLAVE:}

Ecología, aguas volcánicas, fitoplancton, ecosistema mesotrófico, volcán Puracé.

\section{COMPOSITION, DYNAMIC AND ABUNDANCE OF THE PHYTOPLANKTON COMMUNITY IN A LAKE WITH WATER OF VOLCANIC ORIGIN}

\begin{abstract}
The composition and dynamics of phytoplankton in the Aguas Tibias lake , Coconuco, Puracé, is presented in this article. Monthly sampling was performed from October 2006 to June 2007. Temperature, pH, dissolved oxygen, conductivity and nutriments $(\mathrm{N}$ and $\mathrm{P})$ were measured. Phytoplankton was identified and quantified. Fifty-five genera were identified being Diatomophytas the dominant group, followed in importance by Cyanophyta, and Zygophyta Euchlorophyta. In the Aguas Tibias lake the Tabellaria (19.25\%), Anomoeoneis $(10.45 \%)$, Navicula $(7.73 \%)$ and Chlamydomonas $(7.33 \%)$ genera were the most abundant in the water column, compared with the Selenastrum $(0.06 \%)$, Gyrosigma $(0.01 \%)$, and Xantidium $(0.02 \%)$ genera which showed low presence. The variations detected are not statistically significant, both in terms of space (depth) and time (sampling months ). Very high values for calcium, alkalinity, calcic and total hardness and electric conductivity, due, in part, to the volcanic origin of the waterbody. Based on the kinds of algae found, the lake is proposed to be recognized as a mesotrophic ecosystem.
\end{abstract}




\section{KEY WORDS:}

Ecology, waters of volcanic origin, phytoplankton, mesotrophic ecosystem, Puracé volcano.

\section{INTRODUCCIÓN}

La actividad volcánica ha jugado un papel importante en la formación de lagos y lagunas en varias regiones del mundo, como es el caso de los cuerpos de agua en áreas de actividad volcánica o tectónica localizados a lo largo de la Cordillera de los Andes, como la laguna Aguas Tibias que hace parte de esta cadena volcánica en la Cordillera Central colombiana (Roldán, 1992). En Colombia, las investigaciónes biológicas con aguas termales de origen volcánico son escasas. Para la laguna Aguas Tibias se encontró el trabajo de Escobar (2004), en el cual la autora realizó un estudio preliminar de las características físicas, químicas, determinación de las comunidades productoras y la producción primaria, encontrando un ecosistema en condiciones extremas, por los parámetros analizados en este sistema. Los datos más sobresalientes fueron: el pH entre 8,0 y 8,6 , conductividad que osciló entre 790 y $900 \mu \mathrm{mhos} / \mathrm{cm}$, valores de clorofila a entre los 600 y $620 \mathrm{mg} / \mathrm{m} 3$ y una biomasa entre 40.200 y $41.500 \mathrm{mg} / \mathrm{m} 3$ para dos sitios de muestreo. Teniendo en cuenta estos y otros parámetros tomados en los sitos de muestreo, demostró que era un sistema eutrófizado. $\mathrm{Y}$ teniendo en cuenta que las comunidades planctónicas juegan un rol fundamental en la ecología de los cuerpos de agua lénticos y en los lóticos de reducida velocidad, entre las que podemos destacar están: las microalgas conocidas por sus efectos sobre la dinámica de los gases disueltos y los nutrientes, así como su producción acuática (Margalef, 1983; Reynolds, 1997; Umaña-Villalobos, 2010).

La laguna Aguas Tibias es un cuerpo de agua alimentado por una fuente de agua termal que proviene del volcán Puracé. Este sistema se considera de "condiciones extremas" por sus características que no se ajustan a las condiciones de otros cuerpos de agua lénticos de zonas andinas en Colombia. El presente estudio pretende contribuir de manera preliminar al conocimiento, el comportamiento de la composición, abundancia de las comunidades productoras en el ecosistema acuático alto andino laguna Aguas Tibias, localizado en el corregimiento de Coconuco, municipio de Puracé (Cauca).

\section{MATERIALES Y MÉTODOS}

\section{Área de estudio}

La laguna Aguas Tibias está localizada en el municipio de Puracé, corregimiento de Coconuco, ubicado en la zona centro-oriental del departamento del Cauca, sobre la Cordillera Central de los Andes, la cual hace parte del área del Macizo Colombiano, a 02018'38" latitud Norte y 76030'75" longitud Oeste (P.O.T., 2002-2011). Una característica de la laguna Aguas Tibias, es poseer su origen en una fuente de aguas termales provenientes del volcán Puracé, a una altitud de $2.650 \mathrm{msnm}$ y al noroccidente a $12,6 \mathrm{~km}$ del cráter del volcán. La laguna presenta dos entradas de agua, temperatura hídrica promedio de $27^{\circ} \mathrm{C}$, profundidad media de 2,3 $\mathrm{m}$ y régimen de lluvias bimodal. De acuerdo con los registros pluviométricos (20 años) la precipitación promedio es de $1904 \mathrm{~mm}$ anuales. En el primer semestre del año, el periodo de mayores lluvias 
correspondió a los meses de enero, marzo y abril (212,98 mm promedio trimestre). En el segundo semestre del año la mayor precipitación se presentó en los meses de septiembre, octubre, noviembre y diciembre (291,52 mm promedio para los cuatro meses). Los periodos con tendencia seca se presentan en febrero, mayo, junio, julio y agosto $(54,47 \mathrm{~mm}$ promedio para los cinco meses) (IGAC, 1977-1984). En la laguna se observan grandes floraciones algales, las cuales son removidas al vaciar gran parte del agua en algunas ocasiones. La laguna se encuentra en la zona de vida bosque muy húmedo montano bajo (bmh-MB) (Holdridge, 1978). La vegetación ribereña es principalmente herbácea, entre ellas: Nasturtium officinale (berros), Eleocharis sp. (gramínea), Equisetum bogotense (cola de caballo) y Pennisetum clandestinum (kikuyo). La laguna presenta dos entradas de agua que aportan un caudal promedio de $8,8 \mathrm{~L} / \mathrm{s}$. El sustrato es arcilloso. La fauna acompañante son principalmente coleópteros, lepidópteros, dípteros y los peces Poecilia reticulata (guppys) (Longo, 2004).

\section{Sitios y fechas de muestreo}

Se realizaron muestreos entre los meses de octubre de 2006 a abril de 2007. El sitio de muestreo se ubicó en el centro de la laguna, evitando potenciales interferencias en los muestreos por efectos de las corrientes generadas por la entrada de agua. Las muestras para los diversos análisis se tomaron para tres diferentes profundidades de penetración lumínica: $1 \%, 50 \%, 100 \%$, determinada mediante disco Secchi y la aplicación de la fórmula:

\% Penetración lumínica $=(\operatorname{Ln} F) /(D / 1.7)$

Donde:

Ln: Logaritmo natural.

$F$ : porcentaje de penetración deseado.

$D$ : transparencia disco Secchi en metros.

\section{Variables físicas y químicas}

En cada profundidad se obtuvieron datos de temperatura, salinidad, oxígeno disuelto (OD) y transparencia, de acuerdo con las recomendaciones de APHA et al 1992.

\section{Muestras cualitativas}

La recolección de los organismos fitoplanctónicos se realizó con una red cónica simple con tamaño de poro de $24 \mu \mathrm{m}$, mediante arrastres superficiales circulares (Balech, 1977). Las muestras se preservaron en frascos plásticos con solución transeau, de acuerdo con Pinilla (1995). Para la identificación taxonómica de los organismos se utilizaron los trabajos y las claves taxonómicas de Bicudo y Bicudo (1970), Bourrelly (1970, 1972, 1981), Needham y Needham (1982), Blanco y Sánchez (1984), Hino y Tunisidi (1984), Prescott (1984), Bold y Wynne (1985), Tell y Conforti (1986) y Ramírez (2000), entre otros.

\section{Muestras cuantitativas}

Se tomaron muestras en superficie, en la profundidad del disco Secchi y en la profundidad de compensación (profundidad del disco Secchi multiplicada por 3) (Odum, 1986) entre las 10:00 y las 12:00. La preservación se hizo con lugol concentrado y la cuantificación de los organismos se realizó con el método de concentración por sedimentación en cámaras Utermöhl (Lund et al., 1958). Estas cámaras de sedimentación se dejaron decantar dos horas por cada $\mathrm{ml}$ empleado. Las 
algas sedimentadas se contaron en un microscopio invertido (aumento 10X), y la abundancia se calculó teniendo en cuenta el volumen sedimentado y el número de campos visuales contados. Generalmente se contaron entre 30 y 45 campos por cámara de sedimentación ya que, según Ramírez (1992), este número de campos permite aproximarse al $90 \%$ de los organismos presentes en la superficie de conteo.

\section{Análisis numérico}

Se hicieron análisis de varianza para examinar las diferencias significativas de las características fisicoquímicas del agua entre los muestreos, y se empleó el test de Kruskal-Wallis para determinar los muestreos que fueron diferentes (Ramírez, 1999). Para establecer la similitud entre las estaciones se realizaron análisis de clasificación mediante el índice de Bray-Curtis y la estrategia de agrupamiento por promedio simple (Digby \& Kempton, 1987), a partir de los datos estandarizados de las variables fisicoquímicas y de la diversidad de la comunidad fitoplanctónica. Se determinaron los índices de diversidad de Shannon, de dominancia de Simpson como herramientas para interpretar la relación entre el número de especies y su abundancia (Ludwing \& Reynolds, 1988; Ramírez, 1999; Reynolds, 2006). Los datos de la abundancia del fitoplancton se homogenizaron transformándolos mediante la fórmula Log10 $(n+1)$. Para analizar la variación temporal del fitoplancton, se utilizó el programa BioDiversity Pro (McAleece et al., 1997) con el que se hizo el análisis de agrupamientos. Se elaboró una gráfica bivariada de frecuencia vs. abundancia, con la que se determinaron las especies dominantes (alta frecuencia y alta abundancia), temporales (alta frecuencia y alta abundancia), constantes (alta frecuencia y alta abundancia), durante el periodo de estudio.

\section{RESULTADOS Y DISCUSIÓN}

\section{Ambiente físico y químico}

La temperatura del agua registró un valor promedio de $28,2^{\circ} \mathrm{C}$ en toda la columna de agua, con una mínima de $26,3^{\circ} \mathrm{C}$ y una máxima de $30^{\circ} \mathrm{C}$. Es importante mencionar que no se detectaron variaciones en la temperatura en función de la profundidad para cada día de muestreo. Considerando que se trata de un ecosistema alto andino de origen volcánico las aguas son cálidas. Las variaciones observadas no presentaron significancia estadística y pueden deberse a los efectos combinados del viento, lluvias y corrientes observadas. (Tabla 1).

Tabla 1. Valores de los estadísticos de tendencia central y dispersión de las variables físicas y químicas y de la prueba de Kruskal-Wallis para la variación temporal.

\begin{tabular}{|c|c|c|c|}
\hline Variable & m & $\mathrm{CV}(\%)$ & a \\
\hline Temperatura hídrica ${ }^{\circ} \mathrm{C}$ & 28,2 & 4,4 & $0,014^{*}$ \\
\hline Conductividad eléctrica $\left(\mu \mathrm{mhos} / \mathrm{cm}^{2}\right)$ & 760,5 & 15,2 & $0,005^{* *}$ \\
\hline $\mathrm{pH}$ & 8,1 & 2,7 & $0,017^{*}$ \\
\hline Alcalinidad $\left(\operatorname{mgCaCO} . I^{-1}\right)$ & 328,0 & 65,8 & $0,011^{*}$ \\
\hline Dureza total (mgCaCO $\left.3 . I^{-1}\right)$ & 437,1 & 30,5 & $0,015^{*}$ \\
\hline Nitratos (mg..$^{-1}$ ) & 57,7 & 63,0 & $0,005^{* *}$ \\
\hline Fosfatos (mg..$^{-1}$ ) & $1,56 \times 10^{-4}$ & 79,2 & $0,014^{*}$ \\
\hline
\end{tabular}




$$
\begin{gathered}
\text { n.s. = no significativos }(\alpha>0,05),{ }^{*}=\text { significativo }(\alpha<0,05),{ }^{* *} \text { altamente } \\
\text { significativo }(\alpha<0,01) .
\end{gathered}
$$

La conductividad presentó valores entre 600 y $1000 \mu \mathrm{mhos} / \mathrm{cm}^{2}$, los cuales se consideran altos ecosistemas acuáticos continentales alto andinos (Donato, 2001), ya que el origen volcánico determina aguas cloruradas, sulfatadas y carbonatadas. El valor máximo registrado (1200 $\mu \mathrm{mhos} / \mathrm{cm}^{2}$ ) fue en el mes 1 (octubre, en el fondo) y el mínimo (600 $\mu \mathrm{mhos} / \mathrm{cm}^{2}$ ) en el mes 8 (junio, en las tres profundidades).

$\mathrm{El} \mathrm{pH}$, arrojó valores con una tendencia a la alcalinidad en el sistema oscilando entre 7,8 y 8,5 unidades. El valor mínimo $(7,8)$ se registró en el mes 2 (noviembre 2006, en superficie y fondo) y el máximo $(8,5)$ en los meses 6 y 7 (marzo y mayo 2007, en toda la columna de agua). Se concluye que el $\mathrm{HCO}_{3}$ - presenta su máximo porcentaje y comienzan a cobrar importancia los $\mathrm{CO} 3=$ que en conjunto dan una condición tampón al sistema, ocasionando los altos valores de alcalinidad, dureza total y relativa estabilidad del $\mathrm{pH}$ a lo largo del tiempo $(\mathrm{CV}=2,7 \%)$. Se consideraron altos los valores de pH debido a que, según Kalff (2002), los valores para el neotrópico corresponden a baja alcalinidad y a un promedio de $75 \mathrm{mg}^{-1} \mathrm{I}^{-1}$.

En relación con el gas carbónico disuelto, los valores registrados en los meses 2 y 4 (noviembre 2006 y febrero 2004, en las tres profundidades) fueron los más altos con valores entre 17 y $20 \mathrm{mg} / \mathrm{L}$. Los meses con los valores más bajos fueron el 3 y el 8 (diciembre 2006 y junio 2007, en las tres profundidades), encontrándose valores entre 0 y $3 \mathrm{mg} / \mathrm{L}$. Las fuentes de este gas en el ecosistema son respiración (animal y vegetal), procesos de degradación de materia orgánica, aguas lluvias que arrastren consigo $\mathrm{CO} 2$ de la atmósfera $\mathrm{y}$, en general, reacciones bioquímicas que se manifiestan tanto en la columna como en el sustrato (Vásquez, 2001).

Las sales disueltas y en general los parámetros que definen los procesos de mineralización en aguas, arrojaron resultados muy bajos, de forma que los valores registrados para sulfuros, fosfatos y cloruros fluctuaron entre 0 $0,2 \mathrm{mg} / \mathrm{L}, 0-0,4 \mu \mathrm{g} / \mathrm{L}$ y $28-80 \mathrm{mg} / \mathrm{L}$, respectivamente. Las concentraciones de sulfuros fueron bajas, y su presencia podría deberse a depósitos sulfurosos en el sedimentos (Ramírez \& Alcaraz, 2002). Las concentraciones de cloruros encontradas en el ecosistema, coinciden con los valores reportados con aguas oligotróficas de ríos y lagos alto andinos, ya que tienden a presentar bajas concentraciones de cloruros (menores de 0,01 mg/L) (Vásquez, 2001).

El fósforo registró valores entre 0 y $0,3 \mu \mathrm{mol} \mathrm{L}^{-1}$. Los meses de octubre y noviembre de 2006 y mayo de 2007 muestran valores superiores a 0,02 $\mu \mathrm{mol} \mathrm{L}{ }^{-1}$ (en las tres profundidades). Los meses restantes están entre $0 \mathrm{y}$ $0,02 \mu \mathrm{mol} \mathrm{L} L^{-1}$. Estos valores pueden corresponder a la fracción inorgánica particulada procedente en algunos casos de rocas volcánicas y de los sedimentos aluviales (Esteves, 1988; Gibbs, 1970).

Los valores máximos para la dureza total (185 mg de $\mathrm{CaCO}_{3} / \mathrm{L}$ ) se registraron en el mes 4 (febrero 2007, en zona media) y los mínimos (140 $\mathrm{mg}$ de $\mathrm{CaCO}_{3} / \mathrm{L}$ ) en los meses 1 y 3 (octubre y diciembre 2006, en superficie y zona media), según estos valores las aguas de la laguna se categorizan como aguas duras (Roldán, 1992).

El calcio en los meses 2, 6, 7 y 8 (noviembre 2006, abril, mayo y junio 2007, en superficie) se presentó en mayores concentraciones, con promedio de $32 \mathrm{mg} / \mathrm{L}$. Dado que el calcio presenta valores altos (mayores a $25 \mathrm{mg} / \mathrm{L}$ de $\mathrm{Ca}$ ) para un sistema acuático tropical, se puede decir que 
las aguas de la laguna son muy productivas, según Ohle (1934 citado por Roldán, 1992).

Las formas de nitrógeno muestreadas: nitratos $\left(\mathrm{NO}_{3}-\right)$, nitritos $\left(\mathrm{NO}_{2-}^{-}\right)$y amonio $\left(\mathrm{NH}_{4}+\right)$, presentaron valores entre 10 y $100 \mathrm{mg} / \mathrm{L}, 0$ y $0,07 \mu \mathrm{g} / \mathrm{L}$, y 0 y $0,2 \mu \mathrm{g} / \mathrm{L}$, respectivamente, los cuales indican procesos de degradación de materia orgánica (Wetzel, 1981).

La alcalinidad, así como la dureza total y carbonácea, presentaron valores altos que oscilaron entre $0-620 \mathrm{mg}$ de $\mathrm{CaCO}_{3} / \mathrm{L}, 130-213 \mathrm{mg}$ de $\mathrm{CaCO}_{3} / \mathrm{L}$ y $230-650 \mathrm{mg}$ de $\mathrm{CaCO}_{3} / \mathrm{L}$, respectivamente. Considerando adicionalmente los valores encontrados para otros factores como la conductividad y salinidad, la laguna se puede considerar como un ecosistema en condiciones forzadas (Margalef, 1983).

Sin embargo, la estabilidad en la temperatura, la cual es alta para la zona de estudio, el pH, los altos contenidos de oxígeno, gas carbónico disuelto y la presencia de numerosos iones, favorecen el crecimiento de densas poblaciones de algas y una alta capacidad productiva al ecosistema (Roldán, 1992).

En términos generales, se observa una gran estabilidad en el tiempo en los valores para los diversos parámetros físico-químicos estudiados, con registros completamente diferentes a los de otros ecosistemas lénticos alto andinos que han sido estudiados, como los reportados por Donato (2001). Dado su origen volcánico, las aguas presentan características no esperadas para ecosistemas de alta montaña, donde se presentan valores relativamente bajos para parámetros como la temperatura, el oxígeno disuelto, el gas carbónico, la conductividad y los nutrientes. Los suministros de nutrientes y energía para la laguna son permanentes y abundantes, lo que permite estabilizar el sistema y facilitan el desarrollo de comunidades productoras y consumidoras.

Los análisis estadísticos (ANOVA, Tuckey, Duncan, Krustall-Wallis), para los datos fisicoquímicos, permitieron establecer que las variaciones detectadas no son significativas $(0,19<p<0,97)$, tanto en espacio como en tiempo, concluyendo una gran estabilidad en los parámetros físicos y químicos biológicos.

\section{Riqueza específica del fitoplancton}

Se determinaron 55 géneros pertenecientes a siete divisiones (Cyanophyta, Diatomophyta, Ulothricophyta, Euchlorophyta, Zygophyta, Rhodophyta y Pyrrophyta). La división que presentó mayor variedad de géneros fue Diatomophyta (16 géneros), siguiéndole en importancia Cyanophyta, Euchlorophyta y Zygophyta (13, 9 y 9 géneros, respectivamente). En la Tabla 2 se presenta la lista sistémica de los géneros de la laguna Aguas Tibias. El diagrama de frecuencia vs. Abundancia (Figura 1) mostró que los géneros Tabellaria, Anomoeoneis, Navicula, chlamydomona, Oscillatoria, Merismopedia, Chrococcus, Gomphonema, Melosira y Pseudoanabanena fueron dominantes; 20 géneros se determinaron como constantes, de los cuales Staurastrum sp.2, kirchneriella, Traquelomonas, Pinnularia sp.1 y Crucigenia presentaron densidades importantes. Veintiún géneros fueron raros. 


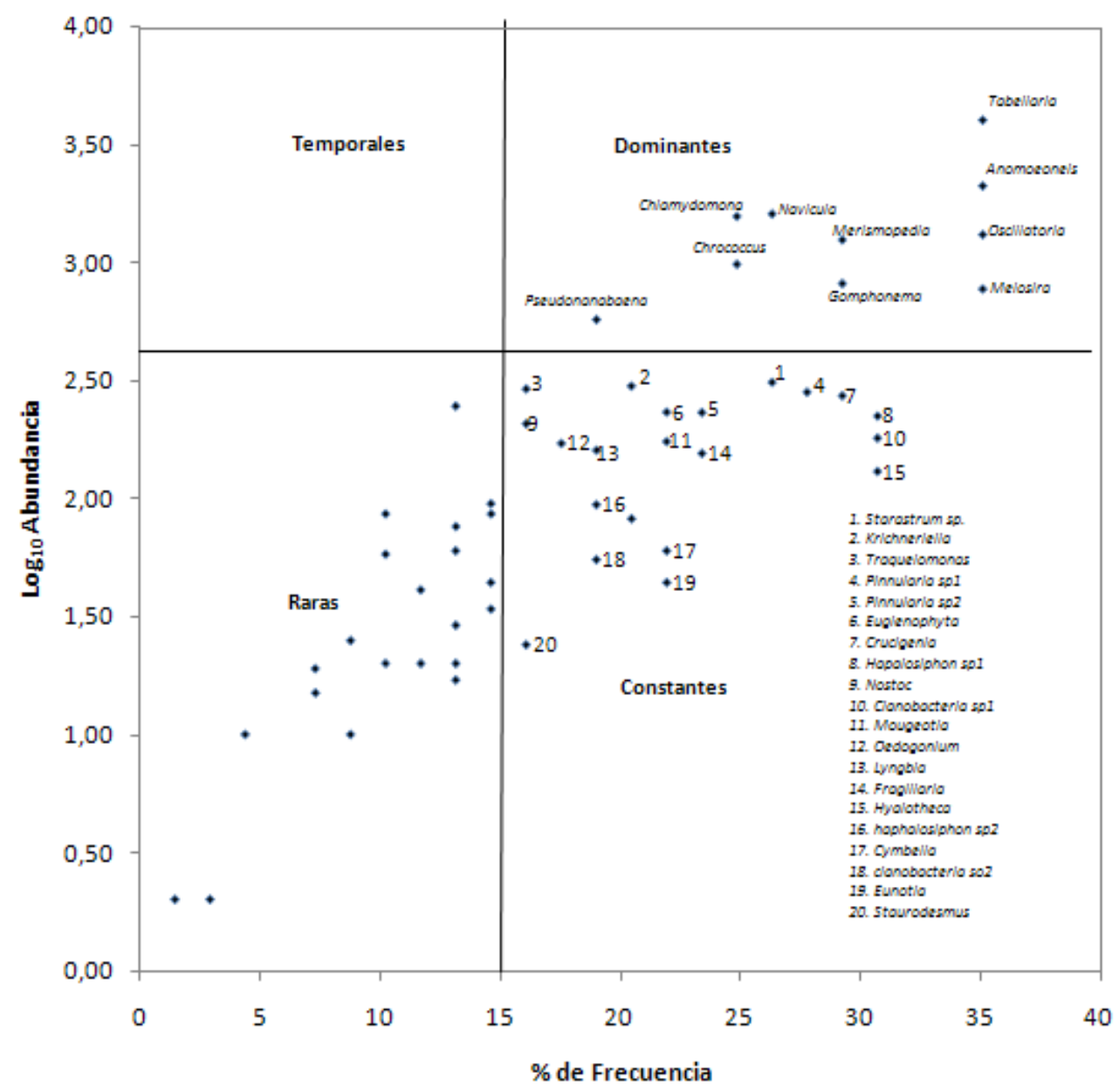

Figura 1. Diagrama de frecuencia vs. Abundancia de la laguna Aguas Tibias.

Tabla 2. Listado de taxa de fitoplancton del la laguna Aguas Tibias durante el periodo octubre 2006 - mayo 2007. $(D=$ Dominantes, $C=$ Constantes, $\mathrm{R}=$ Raras). 


\begin{tabular}{lc}
\hline LISTA DE TAXA & CATEGORIA \\
\hline CLASE: & \\
Cyanophycées & \\
Anabaena & $\mathrm{R}$ \\
Borzia & $\mathrm{R}$ \\
Calothrix & $\mathrm{R}$ \\
Chrococcus & $\mathrm{D}$ \\
Krichneriella & $\mathrm{C}$ \\
Melosira & $\mathrm{D}$ \\
Merismopedia & $\mathrm{D}$ \\
Navicula & $\mathrm{D}$ \\
Oscillatoria & $\mathrm{D}$ \\
Pinnularia sp.1 & $\mathrm{C}$ \\
Pinnularia sp.2 & $\mathrm{C}$ \\
Selenastrum & $\mathrm{R}$ \\
CLASE: & \\
Diatomophycées & \\
Anomoeoneis & $\mathrm{D}$ \\
Cymbella & $\mathrm{C}$ \\
Euglenophyta & $\mathrm{C}$ \\
Eunotia & $\mathrm{C}$ \\
Frustulia & $\mathrm{R}$ \\
Gomphonema & $\mathrm{D}$ \\
Gyrosigma & $\mathrm{R}$ \\
Hapalosiphon sp.1 & $\mathrm{C}$ \\
Hapalosiphon sp.2 & $\mathrm{C}$ \\
Hyalotheca & $\mathrm{C}$ \\
Mougeotia & $\mathrm{C}$ \\
Nostoc & $\mathrm{C}$ \\
Pseudoanabaena & $\mathrm{D}$ \\
Scenedesmus & $\mathrm{R}$ \\
Tetraedrum & $\mathrm{R}$ \\
Traquelomonas & $\mathrm{C}$ \\
\hline &
\end{tabular}

\begin{tabular}{lc}
\hline LISTA DE TAXA & CATEGORIA \\
\hline CLASE: & \\
Euchlorophycées & \\
Chlamydomona & $\mathrm{D}$ \\
Coelastrum & $\mathrm{R}$ \\
Crucigenia & $\mathrm{C}$ \\
Lyngbia & $\mathrm{C}$ \\
Pleurosigma & $\mathrm{R}$ \\
Spirogyra & $\mathrm{R}$ \\
Spirulina & $\mathrm{R}$ \\
Xantidium & $\mathrm{R}$ \\
Fragillaria & $\mathrm{C}$ \\
CLASE: Pyrrophytes & \\
Ceratium & $\mathrm{R}$ \\
Compsopogon & $\mathrm{C}$ \\
CLASE: & \\
Ulothricophycées & \\
Tabellaria sp.1 & $\mathrm{D}$ \\
Tabellaria sp. 2 & $\mathrm{R}$ \\
CLASE: & \\
Zygophycées & \\
Closterium & $\mathrm{R}$ \\
Cosmarium & $\mathrm{R}$ \\
Oedogonium & $\mathrm{C}$ \\
Staurastrum & $\mathrm{R}$ \\
Staurastrum sp.1 & $\mathrm{R}$ \\
staurastrum sp.2 & $\mathrm{C}$ \\
Staurodesmus & $\mathrm{C}$ \\
Synedra & $\mathrm{R}$ \\
Cianobatecrias & \\
Cianobacteria sp.1 & $\mathrm{C}$ \\
Cianobacteria sp.2 & $\mathrm{C}$ \\
Cianobacteria sp.3 & $\mathrm{R}$ \\
Cianobacteria sp.4 & $\mathrm{R}$ \\
\hline &
\end{tabular}

\section{Diversidad y proporcionalidad}

La diversidad de Shannon, calculada para las aguas superficiales en todos los meses, osciló entre 2,04 y 2,8 bits (19 a 28 géneros con mayor probabilidad de salir en los muestreos); y la dominancia de Simpson tuvo estimaciones entre 0,78 y 0,91 (78 a $91 \%$ de probabilidad de extraer dos individuos de la misma especie en un muestreo) (Figura 2). Todos estos índices revelan una comunidad fitoplanctónica muy diversa y de alta proporcionalidad, es decir con baja dominancia de especies. Parecería que la megadiversidad de la laguna se manifiesta también en las microalgas planctónicas, hipótesis que deberá evaluarse en el futuro. Para entender los resultados obtenidos en la laguna Aguas Tibias es muy importante considerar la confluencia de aguas provenientes del volcán Puracé que contribuyen a aumentar la diversidad del fitoplancton de la laguna. Por otra parte, los meses de octubre de 2006 y diciembre de 2006 presentaron las diversidades más altas (Figura 2), comportamiento que coincide con las abundancias fitoplanctónicas más elevadas (Figura 3), posiblemente porque en dichos muestreos se redujo la dominancia de algunas especies de los géneros Tabellaria, Anomoeoneis, Chlamydomona y los otros géneros considerados como constantes y aumentó la proporcionalidad de la comunidad. El índice de Simpson mostró una baja dominancia, coherente con la alta diversidad de Shannon. 


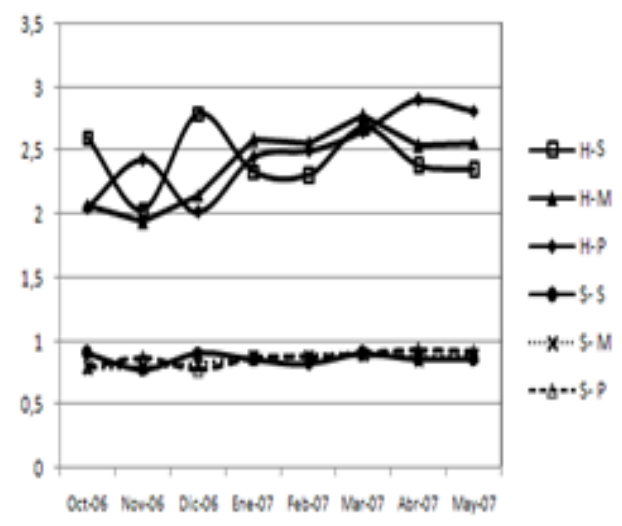

Figura 2. Diversidad de Shannon (H), dominancia de Simpson (S) para el fitoplancton de la laguna Aguas Tibias.

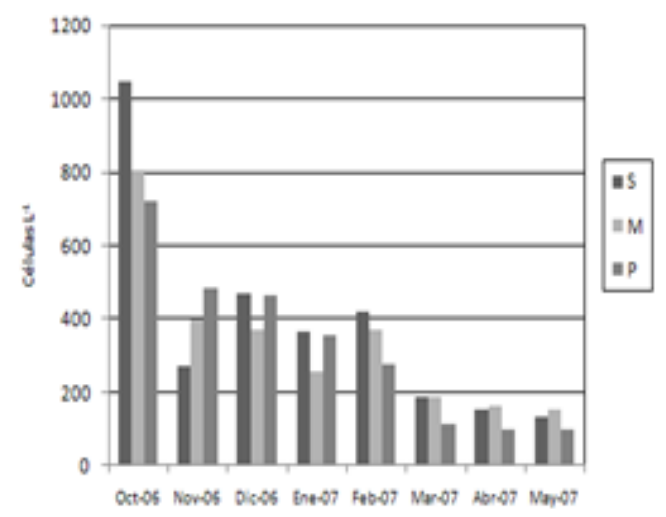

Figura 3. Porcentajes de abundancia de fitoplancton por profundidad $(S=$ superficie, $M=$ profundidad Secchi, $P=$ profundidad de compensación).

\section{Variaciones temporales de la abundancia celular}

Las variaciones en la abundancia de la comunidad fitoplanctónica a lo largo de los muestreos, se deben principalmente a cambios de abundancias de organismos presentes más que a fluctuaciones en el número de géneros. Análisis estadístico, específicamente la prueba Duncan, indica que en los meses 3 y 5 (diciembre 2006 y marzo 2007) se registró mayor número de organismos, en los meses 1, 4, 6 (octubre 2006, febrero y abril 2007) son intermedios y los meses de menor número de organismos son 2, 7, 8 (noviembre 2006, mayo y junio 2007), que concuerda con el vaciado del mes 7. (Figura 3).

Las mayores densidades celulares totales se registraron en el muestreo de octubre de 2006, en el cual la muestra de superficie presentó 1.045,75 células |-1. Por el contrario, en el muestreo de mayo de 2007 se observaron las menores abundancias totales, con valores de 135,22 células ml-1 en superficie (Figura 2). Las abundancias fitoplanctónicas en la laguna Aguas Tibias sugieren un carácter mesotrófico en sus aguas. En cuanto a las diferencias entre las abundancias se observó que las Cianophycées al igual que las Diatomophycées presentaron siempre el mayor porcentaje (Figura 4). 


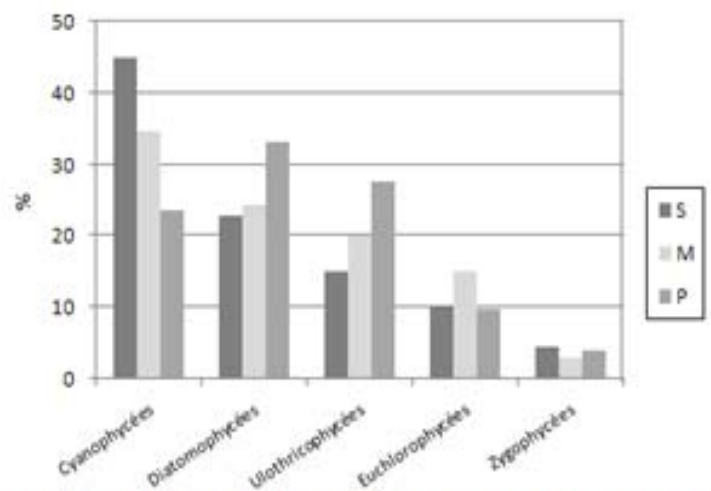

Figura 4. Porcentajes de abundancia del fitoplancton por profundidad ( $S$ = superficie, $M=$ profundidad Secchi, $P=$ profundidad de compensación).

A pesar de que la biomasa algal y la composición específica sean claras indicadoras de cuerpos de agua mesotróficos, no se puede hablar de un claro patrón de sucesión en la laguna, como los que se han reportado para otros lagos (Munawar \& Munawar, 1986; Padisak, 2003). Con la información de diferentes regiones del mundo se han sugerido cuatro tipos de sucesión estacional: I) con dominancia de las cianobacterias; II) con periodos en los que dominan las diatomeas, con dominancia de cianobacterias el resto del tiempo y en algunos casos, con picos cortos de clorococales; III) donde las cianobacterias dominan al final, dominando las clorococales mezcladas con diatomeas y criptofíceas el resto del tiempo; IV) dominancia de poblaciones de flagelados como volvocales y criptofíceas además de las clorococales (Álvarez-Cobelas \& Jacobsen, 1992; Duque, 1996; Ramírez, 2000). La sucesión temporal del fitoplancton observada en la laguna Aguas Tibias, no coincide estrictamente con ninguno de los tipos señalados, aunque tuvo mayor parecido con el tipo II. Inició con el predominio de Cianophycées, el dominio de las Cianophycées continuó hasta el mes de diciembre de 2006 junto con la presencia de Diatomophycées en el mes de febrero de 2007. En el mes de marzo de 2007 se presentó un pulso de Diatomophycées con una disminución de casi todas las demás especies. Este proceso sucesional se hace evidente al analizar la variación en la composición, la riqueza y la densidad fitoplanctónica. Se observa cómo la estructura del ensamblaje algal cambió a través del tiempo, incrementando su diversidad (Figura 2). Cuando los ambientes sufren cambios bruscos, ya sea por las intervenciones antrópicas o propias del sistema, las especies deben usar ambientes diferentes para subsistir e igualmente las perturbaciones interrumpen el curso sucesional normal; por tanto, los procesos en el ecosistema son independientes de las densidades poblacionales (Margalef, 1983). Esto puede ser una explicación a que en los primeros muestreos la densidad algal fuera alta y disminuyera al pasar el tiempo, de manera opuesta a la biomasa y también a la producción. Estas altas densidades iniciales podrían, además, estar relacionadas con resuspensión desde el fondo iluminado o desde la vegetación acuática y de las masas de algas filamentos removidas durante el vaciado y limpieza de la laguna.

\section{Variaciones en la columna de agua}

En la laguna no se encontraron asociaciones estadísticas entre la distribución vertical de las algas y la luz, ya que esta parece ser suficiente y constante en toda la columna de agua, dadas las condiciones de aguas claras y la reducida profundidad de la laguna Aguas Tibias. Los géneros observados están bien adaptados a amplios rangos de radiaciones lumínicas (Reynolds, 1997) y no dependen de la turbulencia para su distribución en la columna de agua, por lo que tienden a tener una 
abundancia equitativa sobre toda la columna (Pinilla, 2006; Canosa \& Pinilla, 2007).

En porcentaje, las Cianophycées al igual que las Diatomophycées fueron siempre las más abundantes en las tres profundidades consideradas (Figura 3). Su dominancia es independiente de la profundidad debido a que están perfectamente adaptadas a vivir en toda la columna de agua. Las grandes ornamentaciones, las largas proyecciones celulares y la tendencia a formar cadenas de células permiten a las Cianophycées y a las Diatomophycées permanecer en las capas superficiales. Las Cianophycées y las Diatomophycées presentaron sus mayores porcentajes de abundancia en el muestreo de octubre de 2006 (30,4\% y $17,3 \%$ en superficie y $15,4 \%$ y $29,5 \%$ en la profundidad Secchi, respectivamente). Según Humm y Wicks (1988) y Reynolds (2006) las cianofíceas prosperan mejor en las capas superficiales que en las profundas, sin embargo, en las aguas de la laguna Aguas Tibias se encontraron representadas en todas las profundidades, en las cuales hay buena disponibilidad de luz.

\section{Análisis de clasificación}

La asociación de los diferentes muestreos, basada en los promedios de las abundancias fitoplanctónicas, se muestra en la Figura 5. La unión de los grupos (con una similaridad mayor al 56\%) estuvo dada por las fechas de muestreo; de esta manera, los muestreos de marzo de 2007 (M6), abril de 2007 (M8) y mayo de 2007 (M3) con los muestreos de enero de 2007 (M4) y febrero de 2007 (M5) se separaron claramente, mientras que el muestreo de octubre de 2006 (M1) y los cuatro muestreos siguientes (M2, M3) se segregaron con menos precisión. Estos resultados, indican que la composición de la comunidad fitoplanctónica fue diferente y característica para cada mes en que se visitó la laguna, en especial para 2006. Dichas diferencias se dieron principalmente a nivel de especies, ya que en conjunto, los grandes grupos de microalgas conservaron una composición semejante durante el periodo de muestreo (Figura 2).

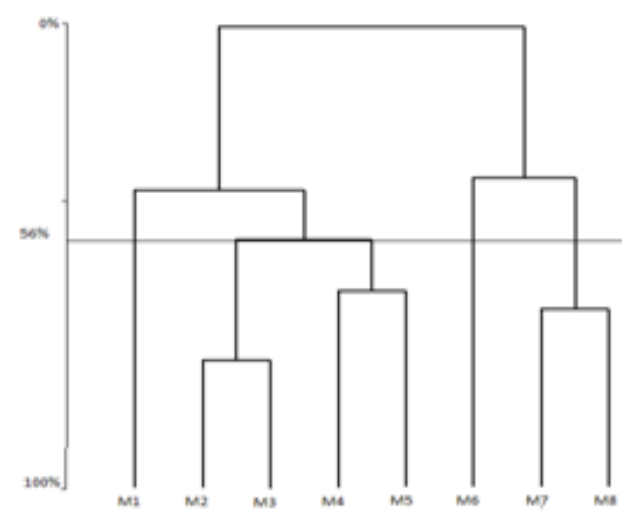

Figura 5. Dendrograma de clasificación de los muestreos de fitoplancton en la laguna Aguas Tibias.

En cuanto a las variables fisicoquímicas evaluadas, en la Tabla 1 se presentan los valores medios, los de dispersión y la significancia de las diferencias entre meses para las variables físicas y químicas analizadas. Con excepción del $\mathrm{pH}$, la temperatura y la conductividad, los coeficientes de variación presentaron valores altos. Entre los meses de muestreo todas las variables mostraron diferencias significativas en la escala de 
tiempo considerada. La temperatura hídrica presentó uniformidad dentro de la columna de agua en cada muestreo. El pH correspondió siempre con condiciones básicas. En la mayoría de aquellos meses en los que no hubo vaciado, la alcalinidad presentó valores altos $\left(328 \mathrm{mgCaCO}_{3} . \mathrm{I}^{-1}\right)$. La dureza total tuvo una tendencia a aumentar de forma semejante al calcio. La conductividad obtuvo los valores más altos en el periodo de invierno $\left(822,5 \mu \mathrm{mhos} / \mathrm{cm}^{2}\right)$. Los valores más bajos de nitratos se dieron en el

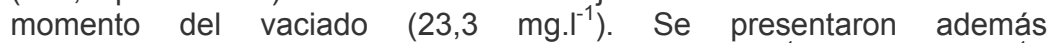
concentraciones muy bajas de fosfatos (entre $0 \mathrm{mg}^{-\mathrm{I}^{-1}}$ y $0,0004 \mathrm{mg} . \mathrm{I}^{-1}$ ); para estos, el registro superior coincidió con el periodo de sequía en mayo, antes de vaciar la laguna $\left(0,0004 \mathrm{mg}^{-1} \mathrm{I}^{-1}\right)$. No se encontraron diferencias significativas entre las profundidades de muestreo, época climática y épocas de vaciado-no vaciado para todas las variables.

En resumen, se puede decir que los cambios fisicoquímicos del agua de la laguna (a excepción del $\mathrm{pH}$, temperatura y conductividad) no fueron muy significativos entre muestreos, pero que a pesar de esto la comunidad fitoplanctónica fue diferente en cada época. Aunque los valores de nitratos de acuerdo con la clasificación de Vollenweider (1968), es un ecosistema que está en el rango de eutrofia. No obstante, los valores de fosfatos de la laguna son muy bajos, posiblemente el nitrógeno y el fósforo disueltos pueden provocar tales cambios en la composición y abundancia del fitoplancton, ya que aumentan el metabolismo de los organismos productores primarios, ocasionando que los ortofosfatos se consuman más rápidamente y en ocasiones se hallen en concentraciones reducidas (Roldán, 1992; Scheffer, 2004), pero dicha influencia deberá evaluarse en trabajos futuros.

\section{CONCLUSIÓN}

Para el periodo de muestreo considerado, la comunidad fitoplanctónica de la ensenada de la laguna Aguas Tibias se caracterizó por la preponderancia de las diatomeas, la baja abundancia de organismos y la elevada diversidad de especies, condiciones propias de ambientes mesotróficos. Las variaciones en la composición de la comunidad ocurrieron principalmente entre muestreos, mientras que los cambios entre profundidades fueron menos intensos. Las variables fisicoquímicas que tuvieron cambios significativos entre muestreos fueron la temperatura, $\mathrm{pH}$ y conductividad. Los análisis clasificatorios confirmaron que la comunidad fitoplanctónica de la laguna fue diferente en cada muestreo, a pesar de que los cambios fisicoquímicos no fueron tan marcados temporalmente, lo cual demuestra que las microalgas son muy sensibles a las condiciones ambientales de la laguna. Se evidenció que el ecosistema reinicia un proceso sucesional en respuesta al disturbio generado por el vaciado y limpieza de la laguna en los primeros muestreos; de tal forma que la variación de la eficiencia fotosintética no es solo afectada por las posibles diferencias en radiación debidas a factores netamente climáticos, sino que en gran parte es un reflejo del cambio sucesional, lo que se evidencia al analizar la disminución en la abundancia algal al tiempo que aumenta la diversidad y se reemplazan algas de la clase Diatomophycée por las de la clase Cianophycée, respectivamente.

\section{AGRADECIMIENTOS}

Este estudio se realizó gracias al apoyo logístico y económico prestado por el Grupo de Recursos Hidrobiológicos de la Universidad del Cauca. Los autores agradecen a todas las personas que brindaron su apoyo en 
esta investigación. Agradecemos igualmente, a los revisores anónimos por sus comentarios y sugerencias, los cuales contribuyeron a mejorar la versión final del manuscrito.

\section{REFERENCIAS BIBLIOGRÁFICAS}

- Álvarez-Covelas, M y B, A. Jacobsen. 1992. Hypertrophic phytoplankton: an overview. Freshwater Forum 2:184-199.

- A.P.H.A.,A.W.W.A \& W.P.C.F. American Public Health Association. 1992. Standard methods for the examination of wáter and wastewater. 18th ed., Washintong, D.C. USA.1268 p.

- Balech, E. (1977). Introducción al fitoplancton marino. Buenos Aires: Eudeba. 211p.

- Bicudo, C. E., y Bicudo, R. M. (1970). Algas de águas continentais brasileiras. São Paulo: Fundação Brasileira para o desenvolvimento o ensino de Ciências.

- Blanco, L., y Sánchez, L. (1984). Contribución al estudio taxonómico de las diatomeas del Orinoco medio, Bajo Caroni y algunas lagunas de inundación (Venezuela). San Félix: Fundación La Salle de Ciencias Naturales.

- Bold, H., y Wynne, M. (1985). Introduction to the algae. 2nd Ed. Englewood Cliffs, NJ: Prentice-Hall Inc.

- Bourrelly, P. (1970). Les algues d'eu douce. Initiation á la systématique. Tome III: Les Algues Bleues et rouges, les Eugléniens, Péridiniens et Cryptomonadines. Paris, Francia: Editions Nouvel Boubée \& Cie.

- (1972). Les algues d'eu douce. Initiation á la systématique. Tome I: Les Algues Vertes. Paris, Francia: Editions Nouvel Boubée \& Cie.

- _ (1981). Les algues d'eu douce. Initiation á la systématique. Tome II: Les Algues Jaunes et Brunes Chrysophycées, Phéophycées, Xanthophycées et Diatomées. Paris, Francia: Editions Nouvel Boubée \& Cie.

- Canosa, A., y Pinilla, G. (2007). Relaciones entre las abundancias del bacterioplancton y del fitoplancton en tres ecosistemas lénticos de los Andes Colombianos. Rev. biol. Trop., 55(1), 135146.

- Digby, P., y Kempton, R. (1987). Multivariate analysis of ecological communities. London: Chapman and Hall. 204p.

- Donato, J. (2001). Fitoplancton de los lagos andinos del norte de Sudamérica (Colombia). Bogotá, D.C.: Academia Colombiana de Ciencias Exactas, Físicas y Naturales. Colección Jorge Álvarez Lleras, No. 19.

- Duque, S. (1996). Caracterización de ecosistemas acuáticos epicontinentales de Colombia con base en el fitoplancton. Bogotá, Colombia: Instituto de Hidrología, Meteorología y Estudios Ambientales (IDEAM). 25p.

- Escobar, A. (2004). Caracterización físico química y determinación de las comunidades productoras y de la productividad primaria de un ecosistema alto andino: Laguna Aguas Tibias - Puracé Cauca. Trabajo de grado para optar al titulo de Bióloga, Universidad del Cauca.

- Esteves, F. A. (1988). Fundamentos de Limnología. 2a ed. Río de Janeiro: Editorial Interciencia. 575p.

- Gibbs, R. (1970). Mechanism controlling world water chemistry. Science, 170, 1088-1090.

- Hino y Tunisidi. 1984. Atlas de algas da represa do Broa. Sao Carlos: Universidade Federal de Sao Carlos. 
- Humm, H., y Wicks, S. (1988). Introduction and guide to the marine blue green algae. New York: John Wiley. 194p.

- IGAC, Subdirección Agrícola. (1977-1984). Zonas de vida o formaciones vegetales de Colombia. 13 volúmenes. Bogotá: Ministerio de Hacienda y Crédito Público.

- Kalff, J. (2002). Limnology. Inland water ecosystems. New Jersey: Prentice-Hall. 592p.

- Longo, M. (2004). Estudio taxonómica y ecológico de Lymnaea (Mollusca: lynaeidae) hospedero intermediario de Fasciola hepática (LINNAEUS, 1758) (TREMATODA: Fasciolidae). Trabajo de grado para optar al título de Bióloga, Universidad del Cauca. 69p.

- Ludwing, J. A., y Reynolds, J. F. (1988). Statistical ecology, a primer on methods and computing. New York: John Wiley. 337p.

- Lund, J. W., Kipling, C., y Lecren, E. D. (1958). The inverted microscope method of estimating algal numbers and the statistical basis of estimations by counting. Hidrobiology, 11, 1-10.

- Margalef, R. (1983). Limnología. Barcelona: Omega. 1010p.

- McAleece, N., Lambshead, P. J. D., Patterson, G. L. J., y Gage, J. D. (1997). BioDiversity Pro. A program for analyzing ecological data. London: Natural History Museum.

- Munawar, M., y Munawar, I. F. (1986). The seasonality of phytoplankton in North American Great Lakes, a comparative synthesis. Hydrobiologia, 138, 85-115.

- Needham, J. G., y Needham, P. R. (1982). Guía para el estudio de los seres de las aguas dulces. Barcelona: Editorial Reverté, S.A.

- Odum, E. P. (1986). Fundamentos de ecología. México: Interamericana. 422p.

- Padisak, J. (2003). Phytoplankton. In O'Sullivan, P. E., y Reynolds, C. S. (Eds.), The lakes handbook. Vol. I: limnology and limnetic ecology (pp. 251-308). Oxford: Blackwell Publishing.

- Pinilla, G. (1995). Ecología regional de pequeños lagos artificiales en el altiplano cundiboyacense. Informe Final. Santafé de Bogotá: Universidad Jorge Tadeo Lozano, Colciencias. 185p.

2006. Evaluación de la eficiencia fotosintética del fitoplancton en un lago amazónico (Lago Boa) y en un lago andino (Lago Guatavita). Acta Amazónica, 36: 221- 228.

- Plan de Ordenamiento Territorial (P.O.T.) para el municipio de Puracé, Cauca 2002-2011.

- Prescott, G.W. 1984. How to know the freshwater algae. 7th Ed. Wm C. Brown Company Publishers, Dubuque, IW, USA

- Ramírez, A. (1999). Ecología aplicada. Diseño y análisis estadístico. Bogotá: Universidad Jorge Tadeo Lozano. 325p.

- Ramírez, J. J. (1992). Contribución al conocimiento ecológico y taxonómico del fitoplancton de algunos cuerpos de agua importantes para el sector eléctrico colombiano. Medellín: FEN, ISA, Universidad de Antioquia, Medellín. 77p.

- _ (2000). Fitoplancton de agua dulce: aspectos ecológicos, taxonómicos y sanitarios. Medellín: Editorial Universidad de Antioquia.

- Ramírez, J y Alcaraz, H. 2002. Dinámica de la producción primaria fitoplanctonica en un sistema eutrófico tropical: Laguna del parque norte, Medellín, Colombia. Caldasia, 24: 411-423.

- Reynolds, C. S. (1997). Vegetation processes in the pelagic: a model for ecosystem theory. Oldendorf/Luhe, Germany: Ecology Institute. 371p.

- _ (2006). Ecology of phytoplankton. Cambridge, UK: Cambridge University Press.

- Roldán, G. (1992). Fundamentos de limnología tropical. Medellín, Colombia: Universidad de Antioquia. 529p. 
- Scheffer, M. (2004). Ecology of shallow lakes. The Netherlands: Kluwer Academic Publishers.

- Tell, G., y Conforti, V. (1986). Bibliotheca Fhycologica: Euglenophytas pigmentadas de la Argentina. Stuttgart: J. Cramer.

- Umaña-Villalobos, G. (2010). Temporal variation of phytoplankton in a small tropical crater lake, Costa Rica. Rev. Biol. Trop., 58(4), 1405-1419.

- Vásquez, G. (2001). Evaluación de la calidad de aguas naturales, significado y alcances en las determinaciones $y$ análisis de parámetros fisicoquímicos y biológicos fundamentales. Producción intelectual Facultad de Ciencias Exactas y de la Educación, Universidad del Cauca, Popayán.

- Vollenweider R. 1968. Scientific Fundamentals of the Eutrophication of Lakes and Flowing Waters, with Particular Reference to Nitrogen and Phosphorus as Factors in Eutrophication. OECD Reporte. p. 250

- Wetzel, R. G. (1981). Limnología. Barcelona: Omega. 679p.

1. Grupo de Estudios en Recursos Hidrobiológicos, Universidad del Cauca. Departamento de Biología, Universidad del Cauca, Popayán, Colombia. 